Pathologe $2016 \cdot 37: 283-284$

DOI 10.1007/s00292-016-0187-3

Online publiziert: 28. Juni 2016

๑) Springer-Verlag Berlin Heidelberg 2016

CrossMark

\section{Wittekind}

Institut für Pathologie, Universitätsklinikum Leipzig, Leipzig, Deutschland

\title{
Allgemeine Aspekte des Tumorgrading
}

\section{Einleitung}

Bereits Rudolf Virchow vermutete einen Zusammenhang zwischen der Gestalt eines Tumors und dem klinischen Verlauf. Die ersten Versuche, mikroskopische Eigenschaften eines Tumors mit der Tumorbiologie und dem klinischen Verhalten in Beziehung zu setzen, datieren wohl auf David Paul von Hansemann zurück [4-7]. Hansemann, ein Schüler von Virchow, untersuchte systematisch den mikroskopischen Aufbau von Tumoren und publizierte in den 1890erJahren über abnorme Mitosefiguren. Das Buch von Hansemann wurde seinerzeit als revolutionär betrachtet, aber durchaus kontrovers bewertet und stimulierte somit viele wissenschaftliche Diskussionen. Ivan Damjanov hat kürzlich geschrieben, dass die exakte mikroskopische Beschreibung der erfassten Tumoren als eine der ersten „Evidenzbasierten“ Vorgehensweisen angesehen werden könnte [3]. In den 1920erJahren publizierte Albert C. Broders, ein Pathologe der Mayoklinik, seine Erfahrungen mit dem Grading von Plattenepithelkarzinomen der Lippen und korrelierte den histologischen Grad mit dem Ausgang der Tumorerkrankung [1, 2]. Er verwendete ein 4-armiges System und klassifizierte Tumoren nach ihrem Gehalt an „inkomplett“ differenzierten Zellen mit einer Unterteilung in 25, 50, 75 oder $100 \%$. Seine Vorstellungen, ein Grading von Tumoren durchzuführen, wurden von vielen anderen Pathologen aufgegriffen und für verschiedene Organtumoren angewandt. Die Ideen von Broders finden sich auch in den heute verwendeten Gradingsystemen wieder. In diese Gradingsysteme wurde unterschiedliche viele histologische
Merkmale aufgenommen, in manchen Systemen bis zu 15 Parameter. In den Gradingsystemen wurden Eigenschaften der Gewebearchitektur eines Tumors ebenso berücksichtig wie zytologische Merkmale. Das Spektrum reichte dabei von der alleinigen Berücksichtigung histoarchitektonischer Parameter beim Grading des Prostatakarzinoms bis hin zu zytologischen, nukleären Gestalteigenschaften beim Nierenzellkarzinom. Das Prinzip, basierend auf den Arbeiten von Broders, war, differenzierte Abschnitte eines malignen Tumors zu identifizieren und die gut differenzierten Abschnitte als Prozentanteile des Gesamttumors anzugeben. Es stellte sich schnell heraus, dass solche Gradingsysteme mühsam in der Anwendung und oft nicht reproduzierbar waren, woraus eine schlechte Korrelation mit der Prognose resultierte. Die Grade wurden in fast allen Arbeiten mit dem 5-Jahres-Überleben korreliert. An ein gutes Gradingsystem wurden deswegen folgende Forderungen gestellt: einfach, leicht anzuwenden, zuverlässig und reproduzierbar. Derartige Systeme sollten in der Lage sein so wurde postuliert - den Ansprüchen längerer Zeiträume zu genügen und sich so langfristig als klinisch nützlich $\mathrm{zu}$ erweisen. Die Gradingsysteme waren nur für die Anwendung an Primärtumoren entwickelt worden. Es wurde versucht, die Kriterien für das Grading des Primärtumors auch für Metastasen oder Tumorrezidive anzuwenden oder sogar für Tumoren, die zuvor mit einer Radio- und/oder Chemotherapie behandelt worden waren. Hinsichtlich der Möglichkeiten der Anwendungen gibt es keinen generell akzeptierten Konsens.

Aufgrund der Defizite aller Gradingsysteme ergab sich die Notwendigkeit, diese im Laufe der Jahre und mit zunehmenden Erfahrungen über fehlende Korrelationen mit der Prognose weiterzuentwickeln. Zu den Weiterentwicklungen gehörten auch Überlegungen die praktisch ausschließlich verwendeten morphologischen Parameter durch zusätzliche, für das Grading wichtige Faktoren zu ergänzen oder zu ersetzen. Viele Parameter wurden ausprobiert, durchgesetzt hat sich bisher nur die immunhistochemische Untersuchung mit dem Antikörper MIB-1 (Ki-67).

Betrachtet man die klinische Bedeutung der Gradingsysteme, dann müssen diese mit den Daten anderer Systeme wie dem Staging und einiger klinischer Scores verglichen und ggf. multivariat analysiert werden. Bei einigen Tumorentitäten wurde das Grading auch bei der Therapieplanung berücksichtigt. Bei niedrig malignen Tumoren ist eine chirurgische Resektion ausreichend, bei hoch malignen Tumoren werden zusätzliche Therapiemaßnahmen gefordert.

Seit fast 100 Jahren sind Gradingsysteme ein wichtiger Bestandteil der pathohistologischen Untersuchung. Sie wurden laufend weiterentwickelt. Die Tatsache, dass sie auch in Zukunft immer weiterentwickelt werden müssen, bezeichnete Damjanov [3] als „Work in Progress“.

Die Beiträge des vorliegenden Heftes haben es sich zur Aufgabe gemacht, den aktuellen Stand des Fortschritts der Gradingsysteme bei verschiedenen Organtumoren aufzuzeigen. Sie werden neben kurzen historischen Rückblicken Feststellungen zu den histologische Subtypen und den Anwendungen von Gradingsystemen machen, die derzeit empfohlenen Gradingsysteme (wenn es mehrere gibt) und die Gradingkriterien beschreiben, die Korrelationen mit der 
Prognose darstellen und Kommentare zu Stärken und Schwächen der vorhandenen Gradingsysteme abgeben.

\section{hitolind}

Prof. Dr. C. Wittekind

\section{Korrespondenzadresse}

\section{Prof. Dr. C. Wittekind}

Institut für Pathologie, Universitätsklinikum Leipzig

Liebigstraße 26, 04103 Leipzig, Deutschland

christian.wittekind@medizin.uni-leipzig.de

Interessenkonflikt. C. Wittekind gibtan, dass er keine finanziellen Verbindungen mit einer Firma hat, deren Produkt in dem Artikel eine wichtige Rolle spielt (oder mit einer Firma, die ein Konkurrenzprodukt vertreibt).

\section{Literatur}

1. Broders AC (1920) Squamous cell epithelioma of the lip: a study of five hundred thirty seven cases. JAMA 74:656-664

2. Broders AC (1926) Carcinoma: Grading and practical applications. Arch Pathol 2:376-381

3. Damjanov I (2013) History and general aspects of grading. In: Damjanov I, Fan F (Hrsg) Cancer Grading Manual, 2. Aufl. Springer, Heidelberg New YorkDordrecht London, S1-7

4. von Hansemann DP (1890) Über asymetrische Zellteilung in Epithelkrebsen und deren biologische Bedeutung. Virchows Arch Pathol Anat Histopath 119:299-326

5. von Hansemann DP (1892) Über die Anaplasie der Geschwulstzellen und die asymetrische Mitose. Virchows Arch Pathol Anat Histopath 121:436-449

6. von Hansemann DP (1897) Die mikroskopische Diagnose der bösartigen Geschwülste. J. A. Hirschwald, Berlin

7. von Hansemann DP (1902) Die mikroskopische Diagnose der bösartigen Geschwülste, 2. Aufl. Hirschwald, Berlin

\section{Molekulares Klassifizierungs- system für Meningeome}

Wissenschaftler des Universitätsklini-

kums Heidelberg und Deutschen Krebsforschungszentrums haben die erste Mutation im Erbgut von Meningeomen entdeckt, die den weiteren Verlauf der Erkrankung entscheidend beeinflusst.

Meningeome entstehen aus der Hirnhaut und machen etwa ein Drittel aller Hirntumoren aus. Diese häufigsten Hirntumoren bei Erwachsenen sind überwiegend gutartig. Allerdings lassen sich aggressiv wachsende Meningeome bisher nicht zuverlässig von gutartigen unterscheiden. Sie werden derzeit u.a. anhand des Erscheinungsbildes ihrer Zellen in die Grade I bis III eingestuft, wobei rund 90 Prozent der Meningeome als gutartig gelten und dem Grad I zugeteilt werden. Sie lassen sich durch eine vollständige operative Entfernung in der Regel heilen. Bei den Graden II und III ist eine anschließende Strahlentherapie angezeigt. Bei derzeit rund 70 Prozent der Patienten mit einem Meningeom des Grades III kehrt der Tumor trotz dieser Behandlung zurück.

Da die histologischen Kriterien, nach denen die Einteilung der einzelnen Tumoren erfolgt, sehr vage definiert sind, ist die Therapie von Meningeom-Patienten bisher problematisch. Hinzu kommen unterschiedliche Ergebnisse, die je nach Prüfer und begutachtetem Bereich der Gewebeprobe variieren. Die Folge sind vermeidbare Rückfälle bei zu harmlos eingestuften und daher fälschlicherweise nicht bestrahlten Tumoren oder unnötige Bestrahlungen im umgekehrten Fall. Außerdem gibt es bisher keine zielgerichteten Therapien bei Tumoren, die nicht chirurgisch entfernt werden können oder erneut heranwachsen. Um diese Herausforderungen anzugehen und zu lösen, erarbeiten die Wissenschaftler aktuell ein komplett neues Klassifizierungssystem, das vollständig auf den molekularen Eigenschaften der Krebszellen basiert. Bestenfalls geben die molekularen Erkennungsmarker der einzelnen Untergruppen bereits wichtige Hinweise auf Schwachstellen der Tumoren und damit neue therapeutische Ziele.
Einige solcher Marker sind bereits bekannt Das reicht für eine neue Einteilung allerdings noch nicht aus. In einigen Meningeomen tritt beispielsweise keine einzige bekannte Mutation auf. Auf der Suche nach neuen Markern haben die Wissenschaftler jedoch kürzlich das Gen TERT (Telomerase reverse Transkriptase) identifiziert, das den Bauplan für ein Protein enthält, welches bei Überaktivierung die Lebensdauer der Krebszellen extrem verlängert. Bei Patienten mit einer solchen Mutation kam es nach der Operation innerhalb eines Jahres zu erneutem Tumorwachstum. Bei unverändertem TERT-Gen liegt die tumorfreie Zeit bei durchschnittlich knapp 15 Jahren. TERT stellt damit einen wichtigen Marker für das neue Klassifizierungssystem dar.

Literatur:

Sahm F, Schrimpf D, Olar A, et al (2016) TERT promoter mutations and risk of recurrence in meningioma. J Natl Cancer Inst 108(5)

Quelle: Universitätsklinikum Heidelberg, www.klinikum.uni-heidelberg.de 